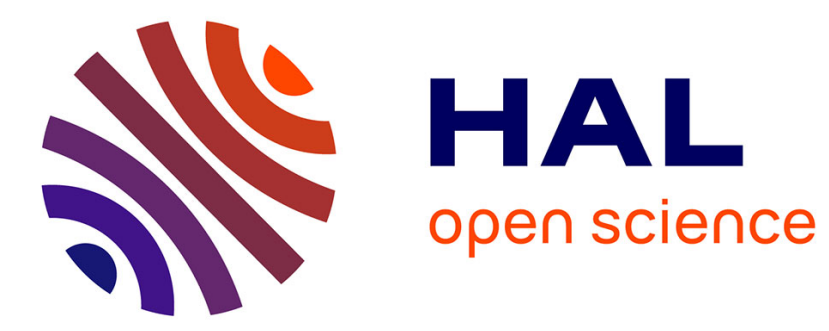

\title{
Telomere-driven mutational processes in yeast
}

Erin Henninger, Maria Teresa Teixeira

\section{To cite this version:}

Erin Henninger, Maria Teresa Teixeira. Telomere-driven mutational processes in yeast. Current Opinion in Genetics and Development, 2020, 60, pp.99-106. 10.1016/j.gde.2020.02.018 . hal-03015050

\section{HAL Id: hal-03015050 https://hal.science/hal-03015050}

Submitted on 19 Nov 2020

HAL is a multi-disciplinary open access archive for the deposit and dissemination of scientific research documents, whether they are published or not. The documents may come from teaching and research institutions in France or abroad, or from public or private research centers.
L'archive ouverte pluridisciplinaire HAL, est destinée au dépôt et à la diffusion de documents scientifiques de niveau recherche, publiés ou non, émanant des établissements d'enseignement et de recherche français ou étrangers, des laboratoires publics ou privés. 


\title{
Telomere-driven mutational processes in yeast
}

\author{
Erin Henninger ${ }^{1}$ and Maria Teresa Teixeira,"* \\ 1 Sorbonne Université, PSL, CNRS, UMR8226, Institut de Biologie Physico-Chimique, Laboratoire de Biologie \\ Moléculaire et Cellulaire des Eucaryotes, F-75005 Paris, France \\ * correspondence to Teresa.teixeira@ibpc.fr
}

\section{ABSTRACT}

Telomeres are part of the system that guards genome integrity in eukaryotes, protecting linear chromosomes from fusions and degradations. The protective functions of telomeres are put at risk in physiological situations where telomeres shorten and trigger replicative senescence. Current models suggest that when telomeres shorten, combined actions of the DNA damage signaling network, DNA repair pathways, and the mechanics of mitosis result in translocations, gene losses, and aneuploidy. In yeasts, many of these processes (signaling, repair, mitosis) can be molecularly dissected because telomerase can be experimentally removed to enable detection of early and rare events. Here we review recent findings on telomere-driven mutational processes in yeast models and discuss how telomere dynamics may contribute to genome evolution.

\section{MAIN TEXT}

\section{Introduction}

Telomeres help maintain genome integrity by preventing chromosome ends from being recognized and processed as accidental chromosomal breaks [1]. Telomeres have several functions, including maintaining their own length by regulating the telomerase reverse transcriptase. Telomerase activity is down-regulated in many human somatic cells, causing telomeres to progressively shorten $[2,3]$. Below a critical length, telomeres activate the DNA damage checkpoint, causing cells to enter replicative senescence or apoptosis [4-6]. During this process, rare cells are subject to genome instability, an alternative cell fate we examine here.

Critically short telomeres have two major consequences. First, they limit cell division, organ regeneration and cancer cell proliferation; critically short telomeres act as tumor suppressors but can also cause lethal telomeropathies $[7,8]$. Second, critically short telomeres can trigger genome instability, and perhaps result in cancer [9-11]. Thus, telomere shortening generally suppresses oncogenesis by limiting cell division, but also promotes cancer by initiating occasional but impactful genome instability. In this review, we highlight recent studies in yeasts that shed light on the origins of the rare initial events that place telomeres at the cross roads of chromosome integrity and evolution. We will focus on events occurring before or at senescence that are caused by telomerase depletion. Readers can refer to an excellent recent review on post-senescence survival mechanisms [12].

\section{Telomere maintenance defects lead to a gradient of genome instability from chromosome ends to interiors}

Saccharomyces cerevisiae, Schizzosaccharomyces pombe, and other related yeasts have contributed tremendous knowledge to our current understanding of telomere biology. Much of the history of telomere discovery is derived from studies in yeasts, including their evolutionary conservation and major functions (Fig. 1). Also, the power of yeast genetics enables detection of rare events in a large population of cells, and enables the description of genome plasticity after inactivation of telomerase.

In budding yeast, telomerase inactivation leads to progressive telomere shortening and loss of viability. Cell cultures eventually recover to contain post-senescent survivors that maintain telomeres through homology- 
dependent repair mechanisms [12,13]. The first studies on genome instability originating from telomeres used these cultures of telomerase-negative cells to monitor loss of function of reporter genes located at several locations throughout an entire chromosome arm, and allowed estimations of mutation rate per cell division by fluctuation analysis $[14,15]$. These pioneering studies showed that at the senescence peak, mutagenesis near chromosome ends increased about 10-fold, compared to centromere-proximal regions where mutation rates were unchanged. Most mutations were terminal deletions, though other events included point mutations, translocations and chromosome losses. Terminal mutations that arose with shortening telomeres were consistent with a model in which shortened telomeres trigger gross rearrangements at chromosome ends.

End resections and/or telomere fusions as the initial trigger of genome instability in the absence of telomere maintenance.

The next question was to understand the mechanism by which these genome rearrangements occur. An initial idea was that telomere shortening and subsequent deprotection leads to telomere fusions and dicentrics (chromosomes with two centromeres), which would form a bridge between two daughter cells at anaphase if centromeres were pulled in opposite directions. The dicentrics might then break during mitosis and produce new, unprotected DNA ends that could be processed again in the next cell cycle (Breakage-Fusion-bridge cycles, originally described by Barbara McClintock [16]). However, these first studies in budding yeast did not find evidence for dicentrics [15]. Moreover, since genome instability at senescence was decreased in nuclease mutants (EXO1 and RAD1), the authors argued in favour of terminal resections being the initial cause of senescence-related genome instability.

Initial studies of telomerase mutants in fission yeast suggested a different mechanism. In these experiments, telomerase defects frequently led to intrachromosomal fusions of telomeres across the two opposite arms, the result of which was a genome with circular chromosomes [17]. In this species containing only 3 chromosomes, non-lethal intrachromosomal fusions were likely easily selected. Another part of the answer is that non-homologous end joining (NHEJ) is more common in fission yeast than in budding yeast.

A recent study sheds additional light on the origin of the frequent intrachromosomal fusions in S. pombe [18]. A high frequency of intrachromosomal telomere associations is also favoured by subtelomeric sequences immediately adjacent to the telomeres, for a yet unclear reason. One hypothesis is that the two subtelomeres from the same chromosome contain highly homologous sequence blocks in opposite directions and facilitate singlestrand annealing (SSA) and subsequent fusions. Conversely, interchromosomal fusions (and conceivably fusions between sister telomeres) could be infrequent because they require an additional event: the inactivation of one of the two centromeres in the resulting dicentrics. In fission yeast, this likely occurs through dismantling of the centromeric chromatin.

Might a telomerase defect lead to chromosome fusions in budding yeast? Based on recent studies in budding yeast, dicentrics might indeed arise from fusions, but the resulting dicentrics tend to break in the fused telomeric sequences to re-establish the two original chromosomes $[19,20]$. Breakage of dicentrics is directed to telomeric repeats by Rap1, the major telomeric protein in budding yeast (Rap1, Fig. 1c), which prevents the binding of condensin. Instead, condensin binds to other parts of the chromosome and causes them to coil away from the budneck. This coiling towards the two spindle pole bodies leaves the Rap1-enriched telomere repeats trapped at the septum between mother and daughter cell; chromosome breakage occurs by septum formation and thus eliminates the telomere-to-telomere dicentric fusions that arose prior to mitosis [20].

A second reason for having underestimated telomere-to-telomere fusions in budding yeast stems from the fact that most studies have been performed in the presence of fully proficient checkpoints, readily able to eliminate cells that might undergo fusion/bridge/breakage cycles from populations. In fact, in budding yeast, telomerase activity depends on the checkpoint kinase activities of Tel1, and to a minor extent, those of Mec1 [21]. Thus, deletion of both of these checkpoint kinases leads to progressive telomere shortening - similar to telomerase inactivation, except that in addition to the absence of telomere maintenance, the signaling of senescence is abolished [13]. Owing to lack of checkpoints, NHEJ-dependent telomere-telomere fusions and subsequent bridges and breakages may be tolerated by the cell, a situation counteracted when telomerase is simply inactivated [22-24]. 
Recent observations suggest an additional role for checkpoints in the course of senescence, linking histone levels to telomere processing, with predictable consequences for genome stability (as discussed [25]).

First, Rap1 is a telomeric protein involved in direct telomere protection from fusions as noted above, but it also acts as a transcriptional activator and repressor of many non-telomeric genes through nucleosome displacement $[26,27]$. Rap1-dependent nucleosome remodelling is also at work during senescence. As telomeres erode, Rap1 re-localizes from telomeres to new target genes in a Mec1-dependent manner [28]. While the details of how Mec1 influences Rap1 displacement are still unclear, this re-localization to new loci results in global chromatin remodelling through two mechanisms: by repressing transcription of histone genes leading to global histone exhaustion, and by locally depleting histones at new target gene promoters through direct interaction with histones [29].

Second, in a budding yeast mec1 tel1 mutant, the increase in telomere-to-telomere fusions is suppressed if histones are also depleted [23]; histone depleted telomeric chromatin favours processing of telomeres leading to recombination rather than NHEJ. Thus, in the course of telomere shortening, persistent activation of Mec1 and Tel1 modulates subtelomeric histone occupancy, shifting the balance of processing pathways to prevent telomereto-telomere fusions and further genome instability.

Third, in fission yeast, a close relationship also exists between chromatin structure and telomere processing. In this organism, chromatin in subtelomeric regions has a non-canonical structure with irregularly spaced nucleosomes and very low nucleosome abundance [30]. Importantly, the Ccq1 telomeric protein (Fig. 1d) contributes to nucleosome deposition at subtelomeres; ccq1 mutant cells show further depletion of nucleosomes at telomeres compared to wildtype cells, and subsequently display a hyper-recombinogenic phenotype among subtelomeric homologous sequences. Therefore, in both yeasts histone density at chromosome extremities is key to regulating telomere fate [31].

Overall, it is likely that both end resections and fusions occur in the absence of telomerase, although the frequency of fusions may be underestimated. They do occur in the wild: scars of telomere-to-telomere fusions can be detected in some yeast genomes at the origin of chromosome reduction during the evolution of yeasts [32]. So, when fusions do arise, what allows them and other telomere-initiated genome instabilities to be tolerated (and perhaps transmitted to progeny of cells) in the presence of fully proficient DNA damage checkpoint pathways?

\section{Instability driven by telomeres may initiate well before senescence proliferation arrest}

Some answers may come from a search of the telomeric structure that triggers the instability. Critically short telomeres are likely present in the vast majority of cells in a senescing population, yet only a tiny minority of these cells undergo non-lethal rearrangements. Thus, it is possible that telomere dysfunction leading to instability is unrelated to telomere length per se. If, as we suggest, a shortened telomere is not be the immediate precursor of instability, what might be?

One strategy to answer this question leverages the study of single cell lineages (i.e. each consecutive cell division from telomerase inactivation to senescence) using microfluidics devices combined with microscopy $[33,34]$. This unique experimental system allows for the capture of events that are frequent enough at the single cell level to cause instability, but rare at the population level. It was unexpectedly discovered that a substantial proportion of cells did not behave in a manner consistent with canonical telomere shortening-driven signaling; they did not irreversibly stop proliferation by persistent activation of the DNA damage checkpoints. Rather, some cells without telomerase exhibited intermittent periods of cell-cycle arrest at DNA damage checkpoints, followed by resumption of normal cell cycles, and subsequent arrest and terminal senescence. Moreover, following an initial arrest due to the DNA damage checkpoints, cells often bypassed arrest by the process of "adaptation to DNA damage", wherein mitosis proceeded even though DNA damage (at telomeres in this case) remained [35]. This process allowed for the proliferation of cells harbouring chromosome alterations, as measured by fluctuation assays and pulse field gel electrophoresis to detect changes in chromosome size. Thus, the succession of telomeric damage, checkpoint activation and adaptation, is a major mechanism by which cells lacking telomerase activity become genetically unstable and accumulate mutations, but keep both their proliferative capacity and checkpoint 
proficiency in the long-term. Many first reversible arrests occur early after telomerase inactivation, when most telomeres aren't expected to be critically short.

\section{Telomere replication stress as a trigger for telomere-driven genomic instability}

One possible explanation for the early transient cell cycle arrests found during the course of senescence described above is faulty telomere replication; a number of recent studies have linked telomere replication errors to genome instability [36]. Telomere replication has long been suggested to be difficult, meaning that some aspect of telomere biology delays replication fork progression through telomeres. In accordance with this, telomerasenegative budding yeast cells become dependent on DNA repair pathways for survival, and more precisely on pathways responding to replication stress. Interstitial telomeric sequences (ITSs) also cause replication stress and undergo frequent breaks during replication for yet unknown reasons [37]. In yeasts, factors that protect telomere replication include DNA replication and repair pathways, helicases that unwind telomeric secondary structures, regulation of transcription of telomeric non coding RNA TERRA and RNA:DNA hybrids, and telomeric proteins themselves. For instance, in fission yeast, it was known that deletion of either Stn1 or Ten 1 telomeric proteins results in abrupt loss of telomeric sequences, suggesting a replication-associated failure leading to telomere loss. The mechanism of how this loss occurs was uncovered using Stn 1 mutants; experiments revealed that these telomeric proteins do indeed prevent excessive fork stalling in the telomeric and subtelomeric regions $[38,39]$.

Recent work in budding yeast also links a defect in a Cdc13-Stn1-Ten1 telomeric complex to telomere instability [40-42]. A strain containing a dispensable disome chromosome with multiple markers served to select for genomic rearrangements. Cdc13 mutant alleles, which purportedly cause excessive ssDNA exposure at chromosome ends, led to a 30 to 50 -fold increase in frequency of formation of unstable chromosomes, perhaps via the formation of dicentrics. In addition, upon further stress with $\mathrm{HU}$ and MMS (which perturb DNA replication), rearrangements linked to the telomere were enriched. The formation of unstable/dicentric chromosomes was also increased in dominant-negative telomerase mutants, pointing to faulty telomeric replication in the absence of telomerase activity as a cause [40].

To further investigate if instability arises from replication stress at telomeres, the bacterial Tus/Ter replication block was introduced at a unique telomere [43], and loss of a gene marker was linked to this Tus/Ter instability. Types of mutations generated by the Tus/Ter barrier at telomeres included large structural variants, small deletions of less than $1 \mathrm{~kb}$, and terminal deletions. Thus, replication stress at telomeres is sufficient to cause genome rearrangements.

Overall, there are several possible scenarios for telomere-linked genome instability (Fig. 2). In addition to the classical view of critically short telomeres being rarely processed as accidental double strand breaks (DSB) to generate genome instability (Fig. 2, light gray), faulty telomere replication before reaching critical length could also contribute to fixation and proliferation of cells carrying genome variants (Fig. 2 middle gray). In the presence of telomerase, replication through telomeres is relatively error-free, either because the telomere repeat is long enough, because telomerase itself assists replication, or because telomerase rescues telomere breaks through unscheduled processing of stalled replication forks. In the absence of telomerase, telomere replication relies on the robustness of telomeric structure and replication-associated repair factors that somehow assist telomeraseindependent replication. For instance, the telomere binding proteins may prevent hazardous repair pathways from acting at telomeres, or may recruit specific ones to ease the passage of replication fork through telomeric repeats. While problems can arise any time and at any telomere size, perhaps as telomeres erode the correct processing of stalled forks may decrease, and lead to end resection, degradation and fusion. Faulty telomere structures in telomerase mutant cells lead either to cell death or to eventual stabilization by homologous recombination with repetitive sequences at telomeres or subtelomeres. Transient instability of chromosomes may arise even in early divisions in telomerase mutant cells, and the transient instability may involve fusion-bridgebreakage cycles limited to breakage near telomeres, thus restoring linear chromosomes. In this view, telomere damage is not lethal per se as it can lead to adaptation of the DNA damage pathway and resumption of cell division after checkpoint activation, with the opportunity for aberrant repair in subsequent cell divisions. 
Despite the considerable challenges that arise during telomere replication, repair of broken telomeres is often achieved, albeit inexactly, and repair can have beneficial consequences. Here, subtelomeric repeats appear to play an intriguing role. The repair of breaks in subtelomeres, which seemingly arise due to defects in the telomeres, is feasible thanks in part to recombination with gene families having similar sequences. Telomeric heterochromatin has been shown recently to aid in this efficient repair [44]. When comparing budding yeast strains with close species, subtelomeric regions appear to be highly recombinogenic, rearranging at a higher rate than the genome as a whole and accumulating point mutations and indels [45-47].

Transposon mobility and telomeres have an intriguing connection. A fraction of genome instability has been attributed to the mobilization of transposable elements, specifically retrotransposons $[48,49]$. Interestingly, the telomerase enzyme is structurally related to retrotransposon reverse transcriptases [50], and subtelomeric regions contain a higher density of transposable elements than elsewhere in their genomes, suggesting that transposition and telomere biology have an entangled evolutionary path [51]. In budding yeast, increased retrotransposition has been detected in the absence of telomerase, and a role for active retrotransposons has been defined in post-senescent survivor cells, which piggyback with $Y^{\prime}$ subtelomeric elements [52-54]. Moreover, chromosomal analysis of Saccharomyces sensu stricto species showed that translocation breakpoints occur preferentially at Ty transposable elements, indicating that ectopic recombination between these repeated elements actually occurred in evolution [55]. Yet, the mechanisms by which telomeres control transposon mobility and associated genome instability remains unknown.

The high rate of rearrangements in subtelomeres appears to be beneficial to yeasts in the wild, contributing to both their survival and pathogenicity [56-59]. Recent studies have attempted to understand how subtelomeric gene families contribute to plasticity in yeast phenotypes. In Candida albicans, the telomereassociated MED2 gene family encodes components of a transcriptional regulatory complex, and has been used to understand how each individual gene contributes to phenotype through the use of inducible promoters for each gene family member. A wide range of phenotypes are affected upon individual expression of genes, including growth, virulence, cell aggregation, handling of oxidative stress, and pathogen host interactions [56,57].

Importantly, telomere shortening increases the frequency of rearrangements at subtelomeric loci up to 1000- fold, even in cases where telomerase is present but limiting. This supports the attractive "adaptive telomere failure hypothesis", which states that low levels of environmental stress lead to hyperrecombination in the subtelomere, and confer a selective growth advantage [47]. One can even speculate that transient changes to telomere length caused by environmental conditions or genetic contexts $[60,61]$ could accelerate or repress the evolution of subtelomeric sequences. Consistent with this idea, in telomerase-negative fission yeast cells, subtelomeres accumulate rearrangements during quiescence [62].

\section{Perspectives}

To conclude, telomeres have a dual function in genome stability. On one hand they protect chromosome ends from fusions and degradations, yet on the other, telomeres are highly dynamic regions that can mutate and even propel evolution [47]. At this point it's unclear to what extent plasticity is selected for in telomere biology; episodic telomere shortening and telomerase deficiency, perhaps combined with replication fork stalling in telomeres and subtelomeres, may be adaptive. After an initial telomere failure, end resections, fusions or both may occur, and drive genome changes. Future work should reveal all the alternatives that telomeres face step-by-step in the absence of normal maintenance mechanisms by telomerase. In particular, it would be interesting to see what structures block telomere replication and what structures arise from their resolutions.

Recent evolutionary genomics data have revealed the importance of subtelomeres in the generation of genetic novelties across all scales of evolution, as shown in studies of budding yeast genomes from wild isolates to domesticated strains for beer production $[46,63]$. The question that remains is whether telomere plasticity is due to the relative non- lethality of genome rearrangements involving subtelomeres, to the regulated dose of telomere length maintenance upon environmental changes, to subtle chromatin alterations or telomere secondary structure changes that impact the way subtelomeres and telomeres are easily replicated, or a combination of these factors. New tools uniquely available in yeasts, including fully synthetic chromosomes devoid of non-essential 
subtelomeres, repetitive sequences, or even telomeres [17,64-66], will perhaps help determine the role of these sequences in the future.

\section{Figures}

(a)

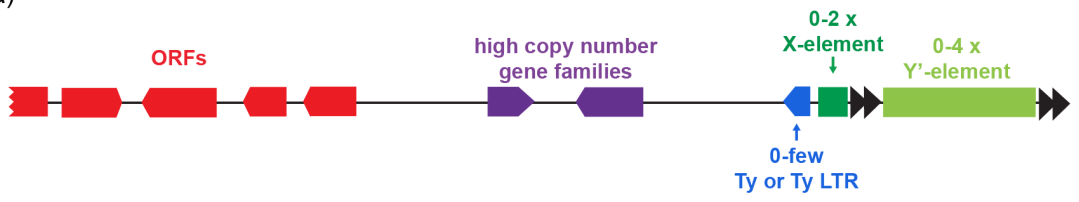

(b) $\quad 7 \mathrm{~L}$ chromosome end

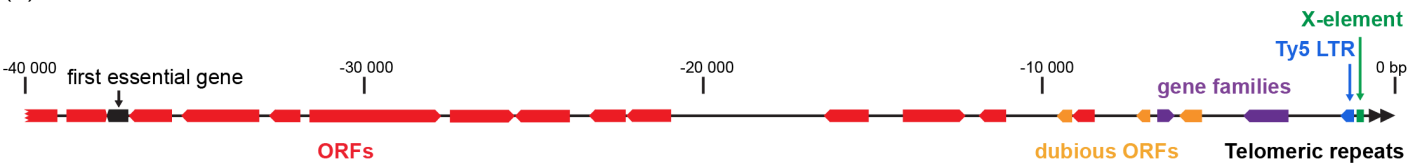

(c) S. cerevisiae

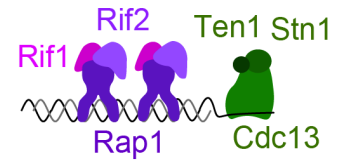

(d) S. pombe

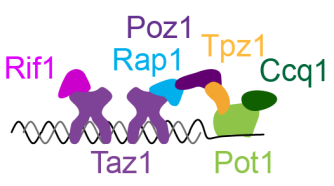

Figure 1: Chromosome ends in model yeasts

(a) Scheme of chromosome ends in budding yeasts, obtained from a recent comparative genomics study of several Saccharomyces species (encompassing different evolutionary scales) using high quality, long-read sequencing data [46]. This study confirms subtelomeres to be generally gene-poor, while still containing non-essential genes families, many of which function to cope with changes in environmental conditions $[67,68]$. In addition, subtelomeric regions may also contain copies of retrotransposons (Ty family), copies of transposon-related subtelomeric $Y^{\prime}$ elements, and copies of X-elements. Overall, subtelomeres are regions of extreme evolutionary plasticity, compared to the core of the chromosomes. (b) Example of $7 \mathrm{~L}$ chromosome end of S. cerevisiae (adapted from [69]). The last $15 \mathrm{~kb}$ of this subtelomere is essential for growth specifically in the absence of telomerase. The mechanism is still unknown. (c-d) Telomeric proteins in S. cerevisiae and S. pombe, respectively. 


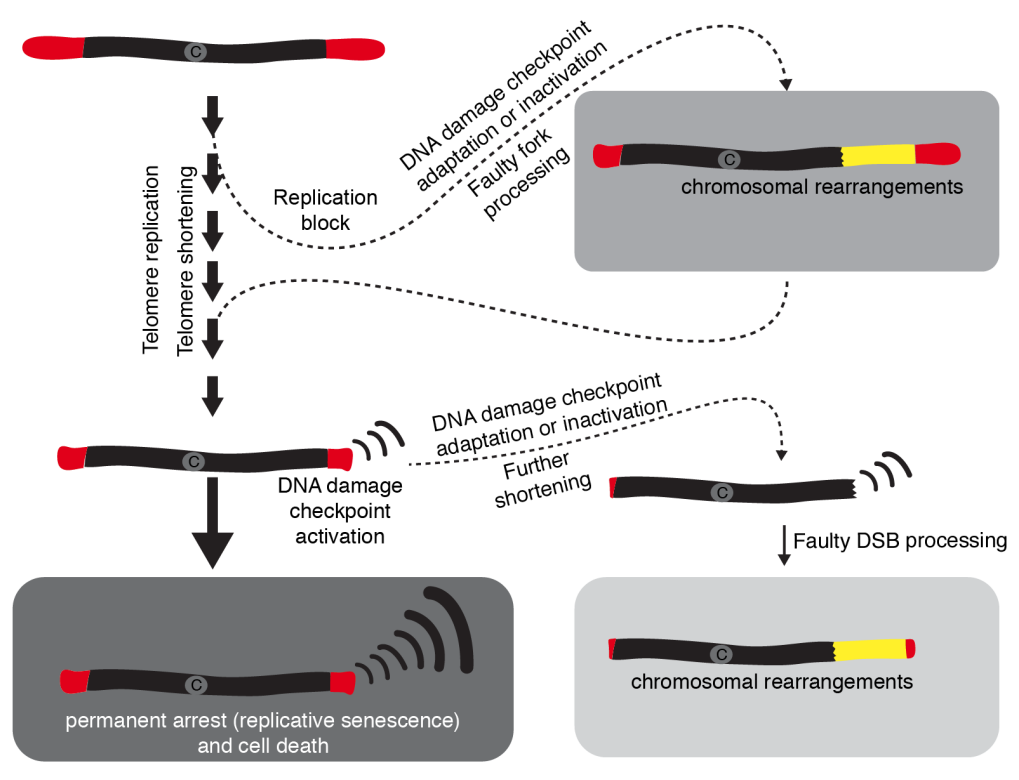

Figure 2: From telomere shortening to chromosome evolution

When telomere length homeostasis is disrupted by experimental depletion of telomerase (or perhaps by an environmental change), the shortest telomere triggers a permanent DNA damage checkpoint activation (dark gray) in the vast majority of cases. However, rare events can cause genome instability. First, as well described in mammalian cells in case of p53 mutations, cells can bypass the senescence limit by checkpoint inactivation, or adaptation, as shown in S. cerevisiae. Telomeres can then further shorten and be subjected to unscheduled DSB processing causing genome rearrangements (light gray). However, evidence from single cell analysis in $S$. cerevisiae shows early events of telomeric damage, DNA damage checkpoint activation and adaptation, linked to genome instability increase. It is well possible that faulty replication fork processing at telomeres initiates genome instability through repair-related processing (medium gray).

\section{Acknowledgements}

We wish to thank Ted Weinert, Rachel Langston and Zhou Xu for the critical reading of the manuscript, as well as the Teixeira lab members for fruitful discussions. We also thank all members of the UMR8226 unit for technical support. Work in MTT lab is supported by the "Fondation de la Recherche Medicale" ("équipe labellisée") and by the French National Research Agency (ANR) as part of the "Investissements d'Avenir" Program (LabEx Dynamo ANR-11-LABX-0011-01) and ANR-16-CE12-0026. EH was supported by a long-term fellowship from the Ligue Contre le Cancer (France).

\section{CREDIT AUTHOR STATEMENT}

Erin Henninger: Conceptualization, Writing - Original Draft, Visualization, Funding acquisition. Maria Teresa Teixeira: Conceptualization, Writing - Review \& Editing, Visualization, Supervision, Funding acquisition

\section{The authors declare no conflict of interest.}




\section{References}

1. Jain D, Cooper JP: Telomeric strategies: means to an end. Annual review of genetics $2010,44: 243-269$.

2. Soudet J, Jolivet $P$, Teixeira MT: Elucidation of the DNA end-replication problem in Saccharomyces cerevisiae. Molecular cell 2014, 53:954-964.

3. Wu RA, Upton HE, Vogan JM, Collins K: Telomerase Mechanism of Telomere Synthesis. Annual review of biochemistry 2017, 86:439-460.

4. Enomoto S, Glowczewski L, Berman J: MEC3, MEC1, and DDC2 are essential components of a telomere checkpoint pathway required for cell cycle arrest during senescence in Saccharomyces cerevisiae. Molecular biology of the cell 2002, 13:2626-2638.

5. Ijpma AS, Greider CW: Short telomeres induce a DNA damage response in Saccharomyces cerevisiae. Molecular biology of the cell 2003, 14:987-1001.

6. d'Adda dFF, Reaper PM, Clay-Farrace L, Fiegler H, Carr P, Von ZT, Saretzki G, Carter NP, Jackson SP: A DNA damage checkpoint response in telomere-initiated senescence. Nature 2003, 426:194-198.

7. Stanley SE, Armanios M: The short and long telomere syndromes: paired paradigms for molecular medicine. Current opinion in genetics \& development 2015, 33:1-9.

8. Artandi SE, DePinho RA: A critical role for telomeres in suppressing and facilitating carcinogenesis. Current opinion in genetics \& development $2000,10: 39-46$

9. Artandi $\mathrm{SE}$, DePinho RA: Telomeres and telomerase in cancer Carcinogenesis 2010, 31:9-18

10. Feldser DM, Hackett JA, Greider CW: Telomere dysfunction and the initiation of genome instability. Nature reviews. Cancer 2003, 3:623627.

11. Maciejowski J, de LT: Telomeres in cancer: tumour suppression and genome instability. Nature reviews. Molecular cell biology 2017, 18:175-186

12. Apte MS, Cooper JP: Life and cancer without telomerase: ALT and other strategies for making sure ends (don't) meet. Crit Rev Biochem Mol Biol 2017, 52:57-73

13. Teixeira MT: Saccharomyces cerevisiae as a Model to Study Replicative Senescence Triggered by Telomere Shortening. Frontiers in oncology 2013, 3:101.

14. Hackett JA, Feldser DM, Greider CW: Telomere dysfunction increase mutation rate and genomic instability. Cell 2001, 106:275-286.

15. Hackett JA, Greider CW: End resection initiates genomic instability in the absence of telomerase. Molecular and cellular biology 2003, 23:84508461.

16. McClintock B: The stability of broken ends of chromosomes in zea mays Genetics 1940, 26:234-282.

17. Nakamura TM, Cooper JP, Cech TR: Two modes of survival of fission yeast without telomerase. Science 1998, 282:493-496.

**18. Tashiro S, Nishihara Y, Kugou K, Ohta K, Kanoh J: Subtelomeres constitute a safeguard for gene expression and chromosome homeostasis. Nucleic Acids Res 2017, 45:10333-10349.

Here the authors succeeded in completely removing subtelomeric homologous sequences from fission yeast to study their function. They show a role for subtelomeres and subtelomeric heterochromatin in telomere fate upon telomerase inactivation.

19. Lopez V, Barinova N, Onishi M, Pobiega S, Pringle JR, Dubrana K, Marcand S: Cytokinesis breaks dicentric chromosomes preferentially at pericentromeric regions and telomere fusions. Genes Dev 2015, 29:322-336.

*20. Guerin TM, Beneut C, Barinova N, Lopez V, Lazar-Stefanita L, Deshayes A, Thierry A, Koszul R, Dubrana K, Marcand S: Condensin-Mediated Chromosome Folding and Internal Telomeres Drive Dicentric Severing by Cytokinesis. Mol Cell 2019, 75:131-144.e133.

Telomere-to-telomere fusions are rarely detected in budding yeast. Here, the authors find the mechanism which counteracts telomere-totelomere fusions: upon their formation, dicentric chromosomes tend to break preferentially at telomeres during cytokinesis due to a differential capacity of condensation of telomeric sequences, reverting the fusions.

21. Ritchie KB, Mallory JC, Petes TD: Interactions of TLC1 (which encodes the RNA subunit of telomerase), TEL1, and MEC1 in regulating telomere length in the yeast Saccharomyces cerevisiae. Molecular \& Cellular Biology 1999, 19:6065-6075.

22. Mieczkowski PA, Mieczkowska JO, Dominska M, Petes TD: Genetic regulation of telomere-telomere fusions in the yeast Saccharomyces cerevisae. Proc Natl Acad Sci U S A 2003, 100:10854-10859.

23. Barrientos-Moreno M, Murillo-Pineda M, Munoz-Cabello AM, Prado F Histone depletion prevents telomere fusions in pre-senescent cells. PLoS Genet 2018, 14:e1007407.

24. Chan SW, Blackburn EH: Telomerase and ATM/Tel1p protect telomeres from nonhomologous end joining. Mol Cell 2003, 11:1379-1387.

25. Prado F, Jimeno-Gonzalez S, Reyes JC: Histone availability as a strategy to control gene expression. RNA Biol 2017, 14:281-286.

26. Pardo B, Marcand S: Rap1 prevents telomere fusions by nonhomologous end joining. Embo J 2005, 24:3117-3127.

27. Mivelaz M, Cao AM, Kubik S, Zencir S, Hovius R, Boichenko I, Stachowicz $A M$, Kurat CF, Shore D, Fierz B: Chromatin Fiber Invasion and Nucleosome Displacement by the Rap1 Transcription Factor. Mol Cell 2019

28. Platt JM, Ryvkin P, Wanat JJ, Donahue G, Ricketts MD, Barrett SP, Waters $H J$, Song S, Chavez A, Abdallah $K O$, et al.: Rap1 relocalization contributes to the chromatin-mediated gene expression profile and pace of cell senescence. Genes \& development 2013, 27:1406-1420.

*29. Song S, Perez JV, Svitko W, Ricketts MD, Dean E, Schultz D, Marmorstein R, Johnson FB: Rap1-mediated nucleosome displacement can regulate gene expression in senescent cells without impacting the pace of senescence. Aging Cell 2019:e13061.

As telomeres erode in the absence of telomerase, Rap1, the major telomeric DNA in budding yeast, delocalizes from telomeres to new genomic promoters to reprogram gene expression. Here, authors demonstrate that Rap1 interacts directly with histone $\mathrm{H} 3 / \mathrm{H} 4$ heterotetramers and causes nucleosome displacement at these promoters.

30. Greenwood J, Patel H, Cech TR, Cooper JP: Fission yeast telosomes: non-canonical histone-containing chromatin structures dependent on shelterin and RNA. Nucleic Acids Res 2018, 46:8865-8875.

31. van Emden TS, Forn M, Forné I, Sarkadi Z, Capella M, Martín Caballero L, Fischer-Burkart S, Brönner C, Simonetta M, Toczyski D, et al.: 
Shelterin and subtelomeric DNA sequences control nucleosome maintenance and genome stability. EMBO reports 2019, 20.

32. Gordon JL, Byrne KP, Wolfe KH: Mechanisms of chromosome number evolution in yeast. PLoS Genet 2011, 7:e1002190.

33. Xie Z, Jay KA, Smith DL, Zhang Y, Liu Z, Zheng J, Tian R, Li H, Blackburn $\mathrm{EH}$ : Early telomerase inactivation accelerates aging independently of telomere length. Cell 2015, 160:928-939.

34. Xu Z, Fallet E, Paoletti C, Fehrmann S, Charvin G, Teixeira MT: Two routes to senescence revealed by real-time analysis of telomerase-negative single lineages. Nature communications 2015, 6:7680.

**35. Coutelier H, Xu Z, Morisse MC, Lhuillier-Akakpo M, Pelet S, Charvin G, Dubrana K, Teixeira MT: Adaptation to DNA damage checkpoint in senescent telomerase-negative cells promotes genome instability Genes Dev 2018, 32:1499-1513.

Microfluidics assays established in budding yeast have enabled to track the consecutive cell divisions from telomerase inactivation to cell death to reveal transient and reversible DNA damage checkpoint-dependent cell cycle delays early before senescence is reached. Here authors demonstrate that many of these events correspond to the process of "adaptation to DNA damage checkpoints", showing that genome instability can indeed initiate early in senescence.

36. Maestroni L, Matmati S, Coulon S: Solving the Telomere Replication Problem. Genes (Basel) 2017, 8

37. Moore A, Dominska M, Greenwell P, Aksenova AY, Mirkin S, Petes T: Genetic Control of Genomic Alterations Induced in Yeast by Interstitial Telomeric Sequences. Genetics 2018, 209:425-438.

38. Matmati S, Vaurs M, Escandell JM, Maestroni L, Nakamura TM, Ferreira MG, Geli V, Coulon S: The fission yeast Stn1-Ten1 complex limits telomerase activity via its SUMO-interacting motif and promotes telomeres replication. Sci Adv 2018, 4:eaar2740.

39. Takikawa M, Tarumoto Y, Ishikawa F: Fission yeast Stn1 is crucial for semiconservative replication at telomeres and subtelomeres. Nucleic Acids Res 2017, 45:1255-1269.

40. Beyer T, Weinert T: Ontogeny of Unstable Chromosomes Generated by Telomere Error in Budding Yeast. PLoS Genet 2016, 12:e1006345.

41. Langston RE, Palazzola D, Bonnell E, Wellinger RJ, Weinert T: Loss of Cdc13 causes genome instability by a deficiency in replicationdependent telomere capping. bioRxiv 2019.

42. Vinton PJ, Weinert T: A Slowed Cell Cycle Stabilizes the Budding Yeast Genome. Genetics 2017, 206:811-828.

**43. Jorgensen SW, Liberti SE, Larsen NB, Lisby M, Mankouri HW, Hickson ID: Esc2 promotes telomere stability in response to DNA replication stress. Nucleic Acids Res 2019, 47:4597-4611.

Here, the authors use a site-specific DNA replication barrier to define the consequences of DNA replication perturbation within a single telomeric locus and recapitulate telomere-driven genome instability initiation.

44. Batte A, Brocas C, Bordelet H, Hocher A, Ruault M, Adjiri A, Taddei A, Dubrana K: Recombination at subtelomeres is regulated by physical distance, double-strand break resection and chromatin status. EMBO 2017, 36:2609-2625

45. Louis EJ, Haber JE: Mitotic recombination among subtelomeric $Y$ repeats in Saccharomyces cerevisiae. Genetics 1990, 124:547-559.

*46. Yue JX, Li J, Aigrain L, Hallin J, Persson K, Oliver K, Bergstrom A, Coupland P, Warringer J, Lagomarsino MC, et al.: Contrasting evolutionary genome dynamics between domesticated and wild yeasts. Nat Genet 2017, 49:913-924

Most recent analysis of subtelomeres in budding yeasts and evolutionary genomics definition of subtelomeric regions.

**47. Mason JMO, McEachern MJ: Mild Telomere Dysfunction as a Force for Altering the Adaptive Potential of Subtelomeric Genes. Genetics 2018, 208:537-548

Using the yeast Kluveromyces lactis as a model, the authors test the prediction of the adaptive telomere failure hypothesis, which suggests that a mild telomere dysfunction could be a driving force of evolution of subtelomeric genes.

48. Anand RP, Tsaponina O, Greenwell PW, Lee CS, Du W, Petes TD, Haber JE: Chromosome rearrangements via template switching between diverged repeated sequences. Genes Dev 2014, 28:2394-2406.

49. Burns KH: Transposable elements in cancer. Nat Rev Cancer 2017 17:415-424.

50. Nakamura TM, Cech TR: Reversing time: origin of telomerase. Cell 1998, 92:587-590.

51. Casacuberta E: Drosophila: Retrotransposons Making up Telomeres. Viruses 2017, 9

52. Maxwell PH, Coombes C, Kenny AE, Lawler JF, Boeke JD, Curcio MJ: Ty1 mobilizes subtelomeric $\mathrm{Y}^{\prime}$ elements in telomerase-negative Saccharomyces cerevisiae survivors. Mol Cell Biol 2004, 24:9887-9898.

53. Scholes DT, Kenny AE, Gamache ER, Mou Z, Curcio MJ: Activation of a LTR-retrotransposon by telomere erosion. Proc Natl Acad Sci U S A 2003, 100:15736-15741.

54. Maxwell PH, Curcio MJ: Incorporation of Y'-Ty1 cDNA Destabilizes Telomeres in Saccharomyces cerevisiae Telomerase-Negative Mutants. Genetics 2008, 179:2313-2317.

55. Fischer G, James SA, Roberts IN, Oliver SG, Louis EJ: Chromosomal evolution in Saccharomyces. Nature 2000, 405:451-454

56. Dunn MJ, Kinney GM, Washington PM, Berman J, Anderson MZ: Functional diversification accompanies gene family expansion of MED2 homologs in Candida albicans. PLoS genetics 2018, 14:e1007326.

57. Flanagan PR, Fletcher J, Boyle H, Sulea R, Moran GP, Sullivan DJ: Expansion of the TLO gene family enhances the virulence of Candida species. PloS One 2018, 13:e0200852.

58. Möller M, Habig M, Freitag M, Stukenbrock EH: Extraordinary Genome Instability and Widespread Chromosome Rearrangements During Vegetative Growth. Genetics 2018, 210:517-529.

59. Ropars J, Maufrais C, Diogo D, Marcet-Houben M, Perin A, Sertour N Mosca K, Permal E, Laval G, Bouchier C, et al.: Gene flow contributes to diversification of the major fungal pathogen Candida albicans. Nature Communications 2018, 9:2253.

60. Romano GH, Harari Y, Yehuda T, Podhorzer A, Rubinstein L, Shamir R, Gottlieb A, Silberberg Y, Pe'er D, Ruppin E, et al.: Environmental stresses disrupt telomere length homeostasis. PLoS Genet 2013, 9:e1003721.

61. Lebel C, Rosonina E, Sealey DC, Pryde F, Lydall D, Maringele L, Harrington LA: Telomere maintenance and survival in saccharomyces cerevisiae in the absence of telomerase and RAD52. Genetics 2009, 182:671-684. 
62. Maestroni L, Audry J, Matmati S, Arcangioli B, Geli V, Coulon S: Eroded telomeres are rearranged in quiescent fission yeast cells through duplications of subtelomeric sequences. Nat Commun 2017, 8:1684.

63. Gallone B, Steensels J, Prahl T, Soriaga L, Saels V, Herrera-Malaver B, Merlevede A, Roncoroni M, Voordeckers K, Miraglia L, et al. Domestication and Divergence of Saccharomyces cerevisiae Beer Yeasts. Cell 2016, 166:1397-1410.e1316.

64. Mercy G, Mozziconacci J, Scolari VF, Yang K, Zhao G, Thierry A, Luo Y, Mitchell LA, Shen M, Shen $Y$, et al.: 3D organization of synthetic and scrambled chromosomes. Science 2017, 355.

65. Pretorius IS, Boeke JD: Yeast 2.0-connecting the dots in the construction of the world's first functional synthetic eukaryotic genome. FEMS Yeast Res 2018, 18.
66. Shao Y, Lu N, Cai C, Zhou F, Wang S, Zhao Z, Zhao G, Zhou JQ, Xue X, Qin Z: A single circular chromosome yeast. Cell Res 2019, 29:87-89.

67. Brown CA, Murray AW, Verstrepen KJ: Rapid expansion and functional divergence of subtelomeric gene families in yeasts. Curr Biol 2010, 20:895-903.

68. Fabre E, Muller H, Therizols P, Lafontaine I, Dujon B, Fairhead C: Comparative genomics in hemiascomycete yeasts: evolution of sex, silencing, and subtelomeres. Mol Biol Evol 2005, 22:856-873.

69. Jolivet P, Serhal K, Graf M, Eberhard S, Xu Z, Luke B, Teixeira MT: A subtelomeric region affects telomerase-negative replicative senescence in Saccharomyces cerevisiae. Scientific Reports 2019, 9:1845. 